\title{
Article \\ Error Analysis and Correction of Thickness Measurement for Transparent Specimens Based on Chromatic Confocal Microscopy with Inclined Illumination
}

\author{
Qing Yu ${ }^{1,2, * \mathbb{D}}$, Chong Wang ${ }^{1}$, Yali Zhang ${ }^{1}$, Shengming $\mathrm{Hu}^{1}$, Ting Liu ${ }^{1,2}$, Fang Cheng ${ }^{1}$, Yin Wang ${ }^{1,2}$, \\ Tianliang Lin ${ }^{1,2}$ and Lin $\mathrm{Xi}^{3}$
}

1 College of Mechanical Engineering and Automation, Huaqiao University, Xiamen 361021, China; 20013080044@stu.hqu.edu.cn (C.W.); 19013080066@stu.hqu.edu.cn (Y.Z.); 1811211007@stu.hqu.edu.cn (S.H.); liut14@hqu.edu.cn (T.L.); chengfang@hqu.edu.cn (F.C.); yin.wangyin@hqu.edu.cn (Y.W.); lt1@hqu.edu.cn (T.L.)

2 Fujian Key Laboratory of Green Intelligent Drive and Transmission for Mobile Machinery, Huaqiao University, Xiamen 361021, China

3 School of Mechanical Engineering, Anhui Polytechnic University, Wuhu 241000, China; eve_q@ahpu.edu.cn

* Correspondence: yuqing@hqu.edu.cn

check for

updates

Citation: Yu, Q.; Wang, C.; Zhang, Y.; Hu, S.; Liu, T.; Cheng, F.; Wang, Y.;

Lin, T.; Xi, L. Error Analysis and Correction of Thickness

Measurement for Transparent

Specimens Based on Chromatic

Confocal Microscopy with Inclined

Illumination. Photonics 2022, 9, 155.

https://doi.org/10.3390/

photonics 9030155

Received: 7 February 2022

Accepted: 3 March 2022

Published: 4 March 2022

Publisher's Note: MDPI stays neutral with regard to jurisdictional claims in published maps and institutional affiliations.

Copyright: (C) 2022 by the authors. Licensee MDPI, Basel, Switzerland. This article is an open access article distributed under the terms and conditions of the Creative Commons Attribution (CC BY) license (https:// creativecommons.org/licenses/by/ $4.0 /)$.

\begin{abstract}
As a fast, high-accuracy and non-contact method, chromatic confocal microscopy is widely used in micro dimensional measurement. In this area, thickness measurement for transparent specimen is one of the typical applications. In conventional coaxial illumination mode, both the illumination and imaging axes are perpendicular to the test specimen. At the same time, there are also geometric measurement limitations in conventional mode. When measuring high-transparency specimen, the energy efficiency will be quite low, and the reflection will be very weak. This limitation will significantly affect the signal-to-noise ratio. The inclined illumination mode is a good solution to overcome this bottleneck, but the thickness results may vary at different axial positions of the sample. In this paper, an error correction method for thickness measurement of transparent samples is proposed. In the authors' work, the error correction model was analyzed and simulated, and the influence caused by the different axial positions of sample could be theoretically eliminated. The experimental results showed that the thickness measurement of the samples was practically usable, and the measurement errors were significantly reduced by less than $2.12 \%$, as compared to the uncorrected system. With this error correction model, the standard deviation had decreased significantly, and the axial measurement accuracy of the system can reach the micron level. Additionally, this model has the same correction effect on the samples with different refractive indexes. Therefore, the system can realize the requirement of measurement at different axial positions.
\end{abstract}

Keywords: chromatic confocal measurement; thickness measurement; error correction; transparent specimen

\section{Introduction}

Chromatic confocal measurement technology is a 3D surface topography measurement technology, which is widely used in many fields such as mechanical manufacturing [1,2], electronic manufacturing $[3,4]$, biomedical $[5,6]$, aerospace $[7,8]$, etc. In order to achieve higher measurement speed, a chromatic confocal method has been proposed. During the measurement process, the height of the measured location will be identified by wavelength from the dispersed light, instead of a position from the physical scanning.

Measurement error analysis and correction is a good research topic in the area of chromatic confocal microscopy. Duque. D [9] et al. studied the dispersion in chromatic confocal microscopy, combining digital image techniques to determine dispersion of translucent materials. Ma et al. [10] improved the dispersion performance by redesigning the dispersion tube lens. Bai J [11] et al. introduced a super-resolving pupil filtering element and 
X-shaped fiber-couple into the chromatic confocal system, which was able to control the peak fitting error within $\pm 0.2 \%$ in a measurement range of $1.05 \mathrm{~mm}$.

Improvement of the data analysis is another direction. Bai et al. [12] proposed a modification to the centroid peak extraction algorithm, where several virtual pixels were interpolated among the real pixels of the spectrometer before thresholding. Chen et al. [13] proposed an error compensation method of peak extraction, which could effectively improve the accuracy of peak extraction. Chen et al. [14] proposed a two-dimensional spectral signal model, which could effectively describe the wavelength shift characteristics. Bai et al. [15] proposed a self-reference dispersion correction to correct the dispersion of the unstable light source or the alterable specimen surface. The results demonstrated that both the robustness and accuracy of chromatic confocal displacement measurement were improved.

In recent years, chromatic confocal technology has also been applied to measure the thickness of transparent specimens. Yu et al. [16] established a thickness measurement model by adding an auxiliary reflector below the specimen. The thickness of the specimen could be determined by comparing the wavelength of light focused on the auxiliary reflector before and after placing the measurement specimen. In 2020, Li et al. [17] proposed an adaptive model decomposition method to separate multiple peaks when they had overlapping areas, to solve a common issue in measuring transparent thin sheets. In 2021, Li et al. [18] proposed a method for measuring the thickness of self-supporting film using double chromatic confocal probes. It skipped the step of calibrating the distance between the starting points of two confocal sensors and could directly obtain the sensor distance through mutual zero measurement.

However, in conventional coaxial illumination mode, both the illumination and imaging axes are perpendicular to the test specimen. When measuring high-transparency specimens, the low signal-to-noise ratio due to low reflectivity of transparent material has not been addressed. By inclining the optical axis, the reflected light will be strengthened while the diffracted light will be weakened [19]. Therefore, the author's team also established a chromatic confocal measurement device with inclined illumination to measure transparent specimens [20]. This declined optical configuration required a separation of illumination and imaging optical paths. This separated configuration may introduce other measurement errors. In this work, it was found that the height change would introduce measurement errors due to model inconsistency. Therefore, in this paper, the key work is to analyze the effect of different axial positions on the thickness measurement error and propose an error correction method to improve the measurement accuracy of an inclined chromatic confocal system.

\section{System Principle and Optical Path Design}

\subsection{Principle of Chromatic Confocal Measurement}

As a non-contact optical measurement method, chromatic confocal technology uses a spectral decoding technology, which is a height/displacement measurement without axial motion, as shown in Figure 1. By establishing the corresponding relation between the axial position of the focal spot and the central wavelength of the focused light, the height/displacement values can be calculated. The schematic diagram of chromatic confocal technology is shown in Figure 2. The polychromatic light source generates multiple wavelength components. The polychromatic light beam passes through a pinhole (pinhole 1 in Figure 2), which modulates the light beam into a point light. Then, the light beam will enter the dispersive tube lens and be distributed along the optical axis according to the different wavelengths. Light beams with different wavelengths will be focused on different positions along the optical axis. Being reflected by the specimen surface, the light beam arrives at the beam splitter and then passes through the detection pinhole (pinhole 2 in Figure 2) which allows only one wavelength to pass through. The height of the specimen surface can be obtained by establishing the correlation between the wavelength of the light that reaches the detector and the axial position of the focal spot. 


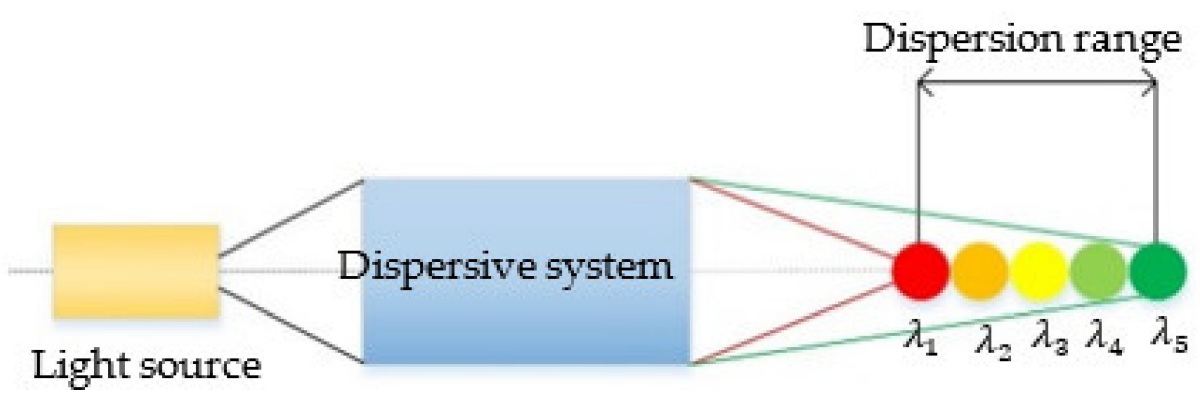

Figure 1. Principle of spectral decoding technology.

\section{Light source}

Pinhole 1

Beam splitter

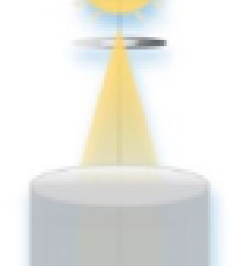

\section{Dispersive tube lens}

Objective

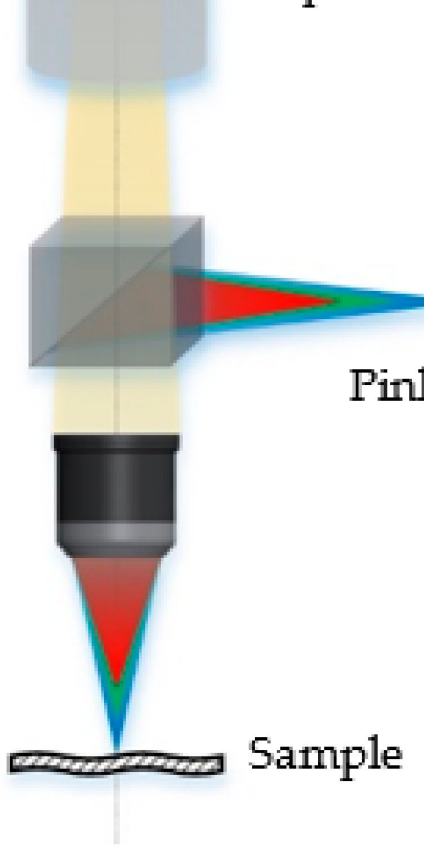

Figure 2. Principle of the chromatic confocal measurement method.

\subsection{Principle of Chromatic Confocal Measurement with Inclined Illumination}

In a conventional chromatic confocal measuring system, the optical axis is set to be perpendicular to the specimen surface. When measuring a transparent specimen surface, it needs to obtain the wavelength values of the light beams which are focused on the upper and lower surfaces, respectively. However, when measuring transparent specimen, the reflecting light intensity will be very weak, as shown in Figure 3. Beam splitters used in such configuration will further lower the effectiveness of energy down to $25 \%$. As a result, the signal-to-noise ratio will be very low. 


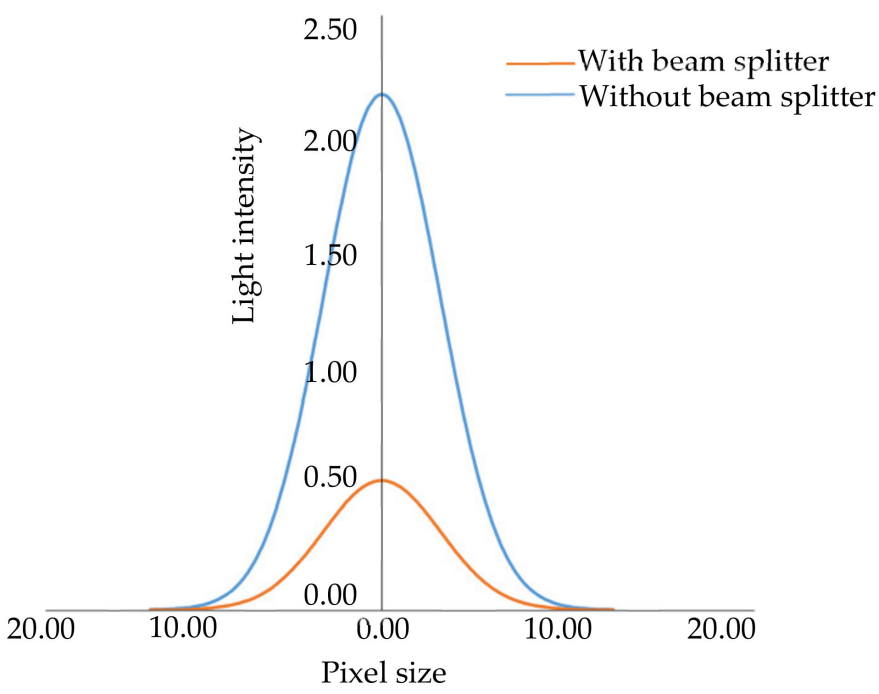

Figure 3. Effect of beam splitter on reflected light intensity.

To solve this problem, an inclined illumination lighting path was developed to the chromatic confocal measurement system, as shown in Figure 4.

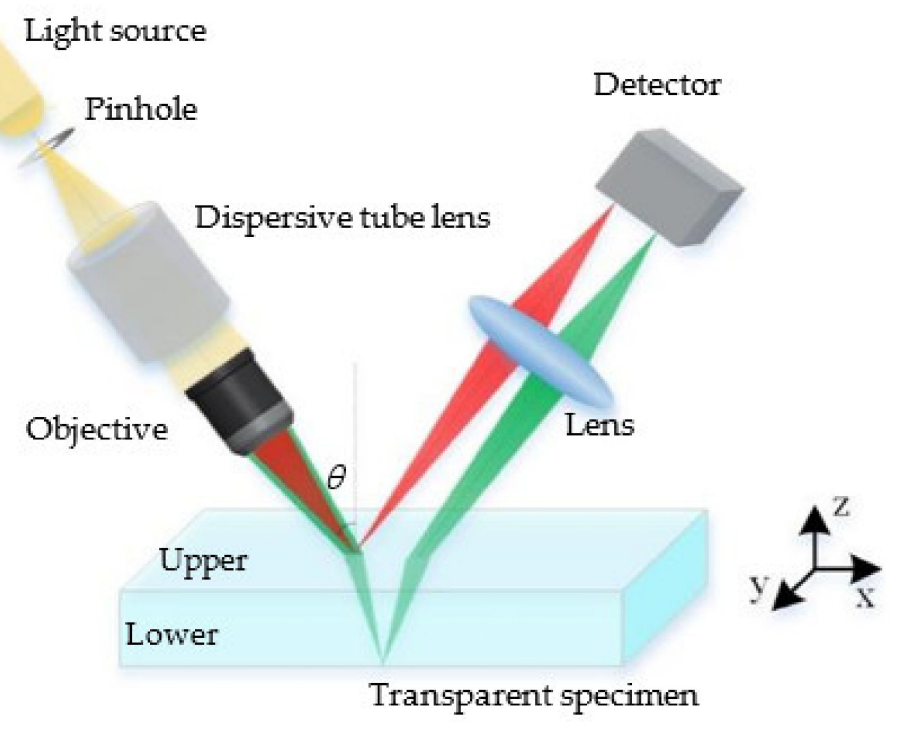

Figure 4. Principle of chromatic confocal with inclined illumination.

The light beams distributed by the dispersive tube lens illuminate the specimen surface with a non-zero incidence angle $\theta$. Compared with the vertical illumination, the extraction of wavelength will be more accurate since the signal-to-noise ratio can be significantly improved.

\subsection{Thickness Calculation Model of Glass Slide}

In a conventional chromatic confocal measurement system (Figure 5), the normalized light intensity at the focal point can be expressed as follows:

$$
I(u)=\left[\frac{\pi a^{2}}{\lambda f} \sin c\left(\frac{u}{4}\right)\right]^{4}
$$

where $u$ is the normalized axial coordinate, $\lambda$ is the wavelength of the incident light, $a$ is the exit pupil radius of the imaging lens, and $f$ represents the focal length of the lens. 


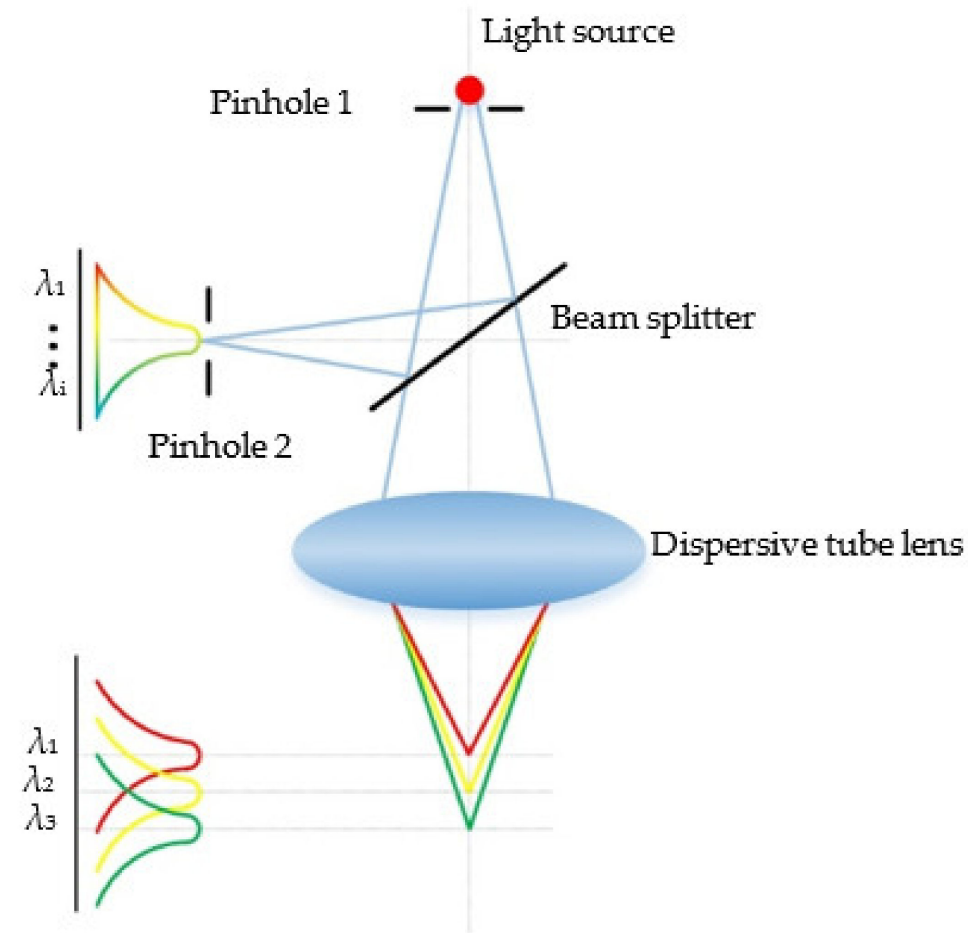

Figure 5. Schematic diagram of the intensity response.

The normalized axial coordinate $u$ can be expressed as follows:

$$
u=\frac{2 \pi}{\lambda} \Delta z \frac{a^{2}}{f^{2}}
$$

In Equation (2), $\Delta z$ is the defocusing amount, which is determined by the response function of axial light intensity in a chromatic confocal measurement system.

In an ideal chromatic confocal system, the axial position has a linear relationship with the wavelength value of the focused light. For a beam of light with a wavelength $\lambda_{\mathrm{i}}$, the defocusing amount is:

$$
\Delta h=f\left(\lambda_{i}\right)-f\left(\lambda_{0}\right)=k\left(\lambda_{\mathrm{i}}-\lambda_{0}\right)
$$

where $f\left(\lambda_{\mathrm{i}}\right)$ represents the optical axial position, $\lambda_{\mathrm{i}}$ represents the wavelength of the focused light.

In Figure 4, the incline angle between the optical axis of illumination path and the normal direction of the specimen surface is defined as $\theta$. Thus, the relationship between $\Delta h$ and $\Delta z\left(\lambda_{\mathrm{i}}\right)$ can be expressed as follows:

$$
\Delta z\left(\lambda_{\mathrm{i}}\right)=\Delta h \cdot \cos \theta
$$

Substituting Equations (3) and (4) into Equation (1), the light intensity of the detector in Figure 4 can be expressed as follows:

$$
I(u)=\left[\frac{\pi a^{2}}{\lambda_{\mathrm{i}}\left(h_{0}+k \lambda_{\mathrm{i}}\right)} \sin c\left(\frac{\pi a^{2} k\left(\lambda_{\mathrm{i}}-\lambda_{0}\right) \cos \theta}{2 \lambda_{\mathrm{i}}\left(h_{0}+k \lambda_{\mathrm{i}}\right)^{2}}\right)\right]^{4}
$$

The light intensity distribution of Equation (4) is simulated, which is shown in the black curve in Figure 6. At the same time, the light intensity distribution curves of different wavelengths are simulated, and it can be found that the black curve consists of all peak points of light intensity at every wavelength. As a result, the chromatic confocal system with inclined illumination also has good spectral frequency selection characteristics. 


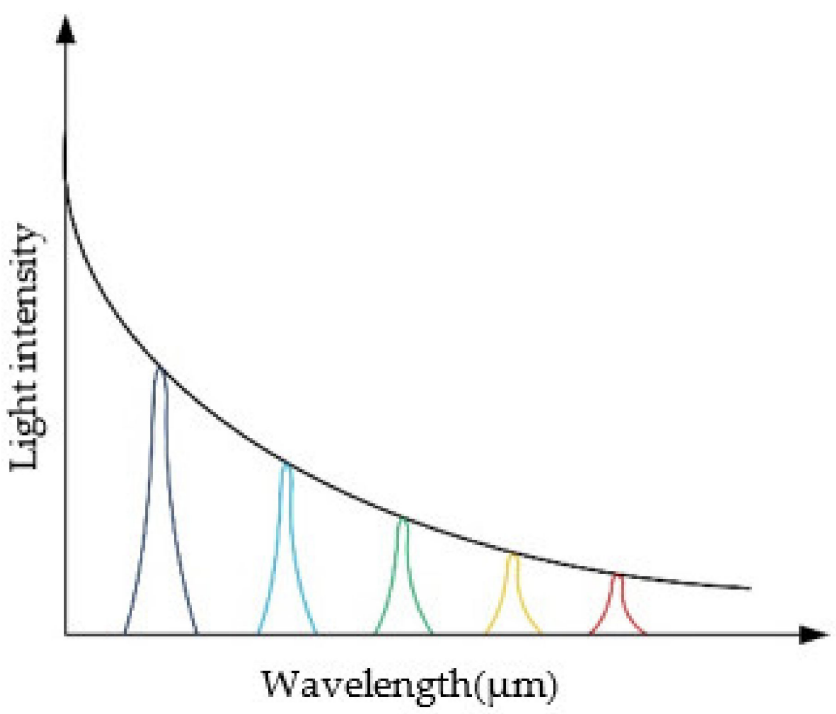

Figure 6. Light intensity distribution with different wavelengths.

Based on this theoretical analysis, the thickness calculation model of a chromatic confocal system with inclined illumination is provided in the following paragraphs.

As shown in Figure 7 , there are two beams of light (wavelength $\lambda_{0}$ and $\lambda_{\mathrm{i}}$ ) focused on the upper and lower surfaces and the focal points are points $O$ and $B$, respectively. If there was no transparent specimen, the light beam with the wavelength $\lambda_{i}$ would be focused on point $C$. However, it is focused on the lower surfaces due to the existence of transparent specimen.

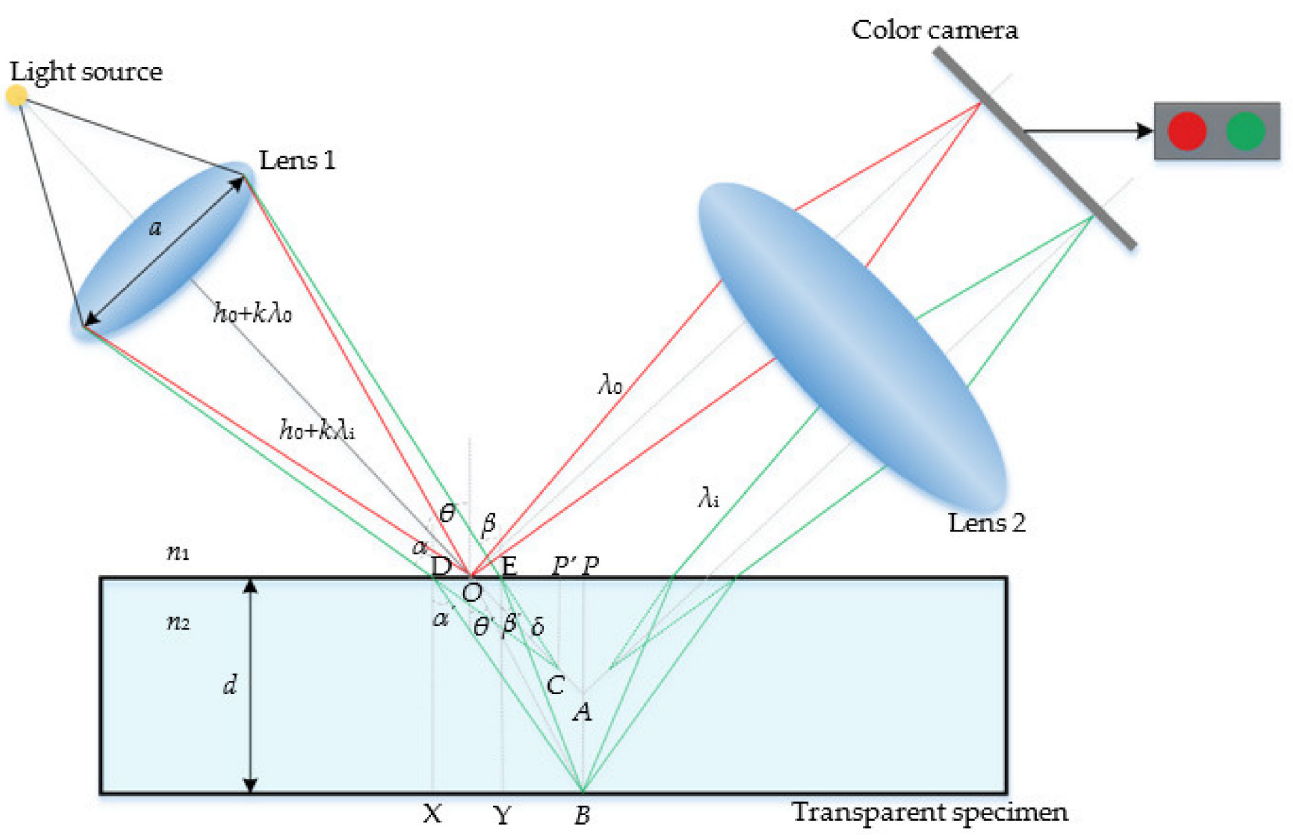

Figure 7. Thickness calculation model of transparent specimen. The letters in the picture represent the following meanings: $n_{1}$ the refractive index of the air; $n_{2}$ : the refractive index of the transparent specimen; $a$ : the diameter of lens $1 ; d$ : the thickness of the transparent specimen; $\theta$ : the inclined angle of the system; $h_{0}+k \lambda_{0}$ : the distance between point $O$ and lens $1 ; h_{0}+k \lambda_{\mathrm{i}}$ : the distance between point $C$ and lens1; $\alpha$ : the index angle of the left light beam with the wavelength $\lambda_{\mathrm{i}} ; \beta$ : the index of the right light beam with the wavelength $\lambda_{\mathrm{i}} ; \alpha^{\prime}$ : the refraction angle of the left light beam with the wavelength $\lambda_{\mathrm{i}} ; \beta^{\prime}$ : the refraction angle of the right light beam with the wavelength $\lambda_{\mathrm{i}}$. 
According to the trigonometric function, the following formula can be obtained:

$$
\begin{aligned}
& \frac{D E}{d}=\frac{X B-Y B}{d}=\tan \alpha^{\prime}-\tan \beta^{\prime} \\
& \frac{D E}{C P^{\prime}}=\frac{D P^{\prime}-E P^{\prime}}{C P^{\prime}}=\tan \alpha-\tan \beta
\end{aligned}
$$

Additionally, the thickness of the transparent specimen $d$ and related parameters can be expressed as:

$$
d=C P^{\prime} \cdot \frac{\tan \alpha-\tan \beta}{\tan \alpha^{\prime}-\tan \beta^{\prime}}=O C \cdot \cos \theta \cdot \frac{\tan \alpha-\tan \beta}{\tan \alpha^{\prime}-\tan \beta^{\prime}}
$$

Substituting Equation (3) into Equation (8),

$$
d=k\left(\lambda_{\mathrm{i}}-\lambda_{0}\right) \cdot \cos \theta \cdot \frac{(\tan \alpha-\tan \beta)}{\left(\tan \alpha^{\prime}-\tan \beta^{\prime}\right)}
$$

$\alpha$ and $\beta$ can be calculated by $\theta$ and $\delta$ :

$$
\left\{\begin{array}{l}
\alpha=\theta+\delta \\
\beta=\theta-\delta
\end{array}\right.
$$

$\delta$ is related to the diameter of the dispersion tube lens and the distance from point $O$ to the dispersion tube lens. In this system, $a$ and $h_{0}$ can represent the two values. Therefore, $\delta$ can be expressed as follows:

$$
\delta=\arctan \left[\frac{a}{2\left(h_{0}+k \lambda_{\mathrm{i}}\right)}\right]
$$

\subsection{Color Conversion Algorithm}

The image shows different colors because it contains $R, G, B$ values. As discussed in the above sections, the axial displacement of the specimen is related to the wavelength value of the focused light. Therefore, it is necessary to convert color information into wavelength information and a conversion algorithm is proposed to convert RGB space into HSI space, where the $H$ value is correlated with the wavelength. So, $H$ value can be directly used to represent wavelength value. The two color spaces are shown in Figure 8.

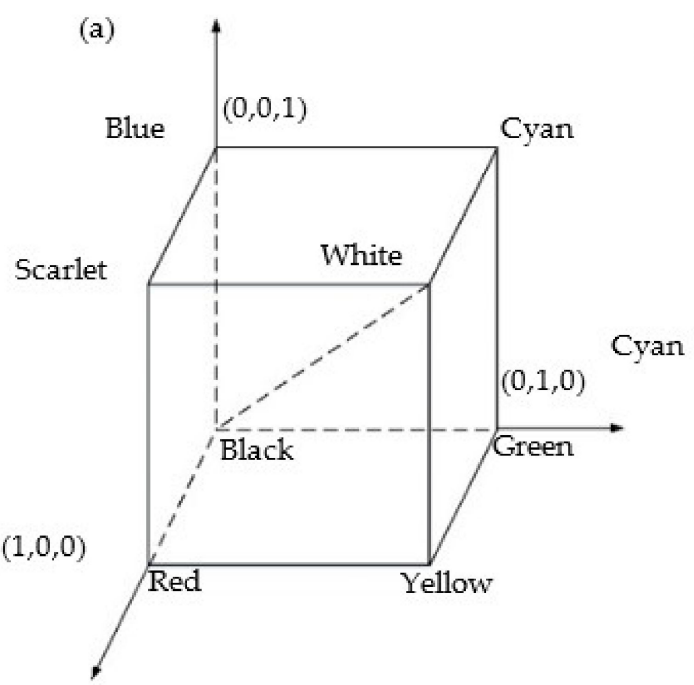

(b) White

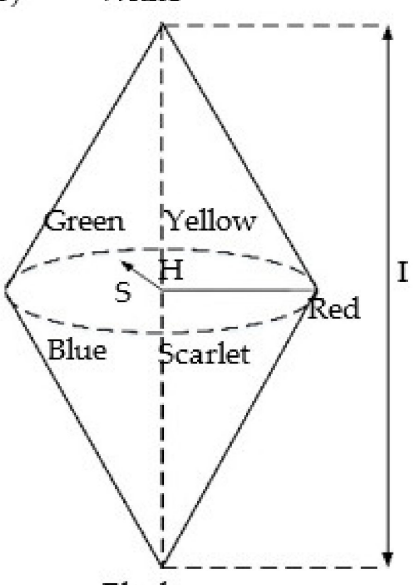

Black

Figure 8. Principle of color conversion algorithm: (a) RGB space; (b) HSI space. 
In the HSI space, $H$ can be expressed as follows:

$$
H= \begin{cases}\theta, & G \geq B \\ 2 \pi-\theta, & G<B\end{cases}
$$

where $\theta$ is expressed as follows:

$$
\theta=\cos ^{-1}\left(\frac{(R-G)+(R-B)}{2 \sqrt{(R-G)^{2}+(R-B)(G-B)}}\right)
$$

It has been proved, theoretically and experimentally, that this color conversion algorithm can achieve micron resolution. Additionally, several articles [20,21] have been published using this color conversion algorithm.

Based on the above, the wavelength can be replaced by $H$ value. Therefore, Equation (3) will become:

$$
\Delta h=k_{1}\left(H_{\mathrm{i}}-H_{0}\right)
$$

Additionally, the thickness of the transparent specimen $d$ becomes the following:

$$
d=k_{1}\left(H_{\mathrm{i}}-H_{0}\right) \cos \theta \frac{(\tan \alpha-\tan \beta)}{\left(\tan \alpha^{\prime}-\tan \beta^{\prime}\right)}
$$

\section{Setup and Calibration Experiment of the System}

\subsection{Setup of the System}

Based on the theoretical analysis mentioned above, a chromatic confocal measurement device with inclined illumination was built, as shown in Figure 9. In this device, the polychromatic light beam was distributed by the self-built dispersive tube lens. The angle $\theta$ was determined by the angle measuring instrument, which was $43^{\circ}$. The transparent specimen was placed on a two-dimensional motion platform which consists of one-dimensional motion platforms and can move in the directions of arrows shown in Figure 9. The color camera was applied as the receiver to obtain the information of focal spots in the reflected light path. The components used in this device are listed in Table 1.

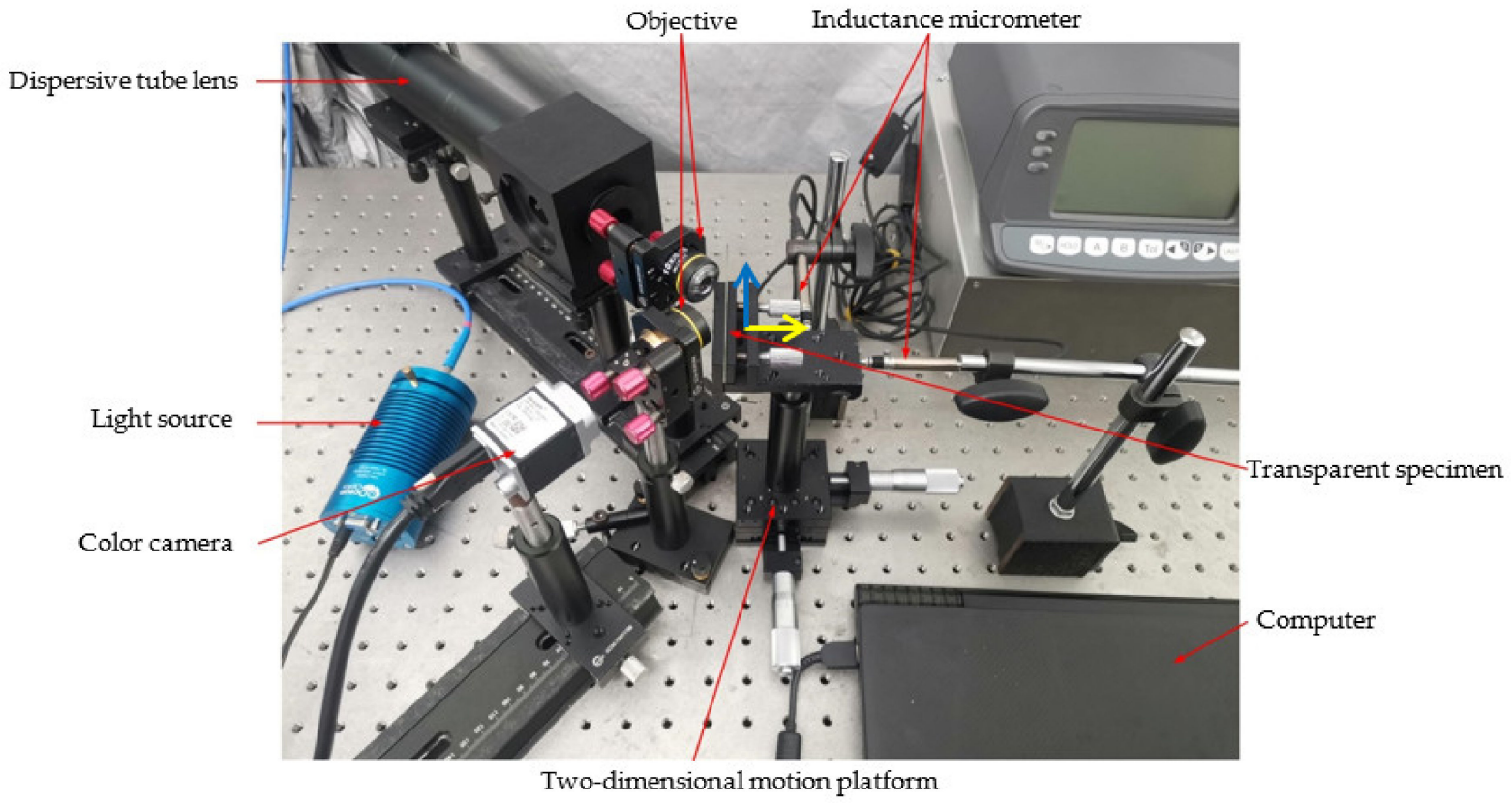

Figure 9. Diagram of chromatic confocal system with inclined illumination. 
Table 1. List of components in the experimental platform.

\begin{tabular}{cc}
\hline Components & Manufacturer \\
\hline Light source & Ocean Optics; HL-2000-FHSA \\
Dispersive tube lens & Self-built \\
Objective & Motic;Magnification:10×, NA value:0.25 \\
Two-dimensional motion platform & Daheng Optics; GCM-T25MC \\
Inductance micrometer & Tesa; TT80 \\
Color camera & Balser; a2A5320-23ucBAS \\
\hline
\end{tabular}

\subsection{Calibration Experiment of the System}

In order to establish the relationship between the position of the specimen surface and the information of the image color, a calibration experiment was performed in this study.

In this experiment, a transparent specimen was chosen as the test sample. The aberrations [22] have been included in the measurement results. Therefore, we treated the aberrations as a systematic error. The sample was moved 23 steps in the direction of the yellow arrow by a two-dimensional motion platform. At the same time, the camera captured 23 color images at different positions and the inductance micrometer recorded the data of each position. The images were converted to $H$ values by the color conversion algorithm. At last, a relationship between $H$ value and the position was established. The interval of the 23 positions was $100 \mu \mathrm{m}$, and the total moving distance was $2200 \mu \mathrm{m}$.

Figure 10 shows the color information of the light spots which focused on the upper and lower surfaces. "The upper" in Figure 10 represents the color change reflected from the upper surface of the transparent specimen. "The lower" in Figure 10 represents the color change reflected from the lower surface of the transparent specimen. From position 1 to position 23, the color of the light spot gradually changed from red to green. In the calibration experiment, only the images from the upper surface of the side were recorded and processed.

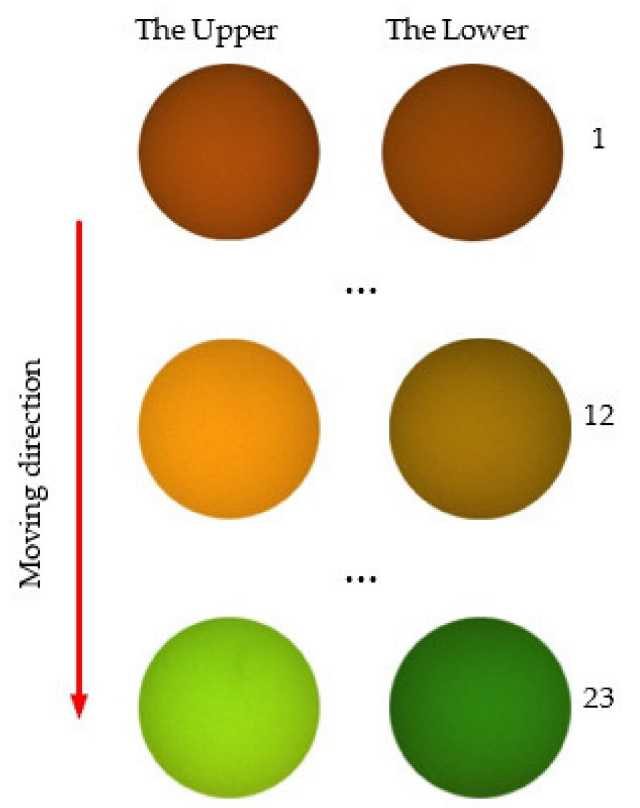

Figure 10. The color change of the calibration experiment.

The $H$ values of 23 focused spots were calculated through color conversion algorithm. Correspond the $H$ value to the data of the positions and note it in Table 2. The calibration curve before fitting is shown in Figure 11a. Within 700-1500 $\mu \mathrm{m}, 9$ points were selected for linear fitting, as shown in Figure 11b. 
Table 2. Calibration result calculated using the date from the upper surface of the sample.

\begin{tabular}{cc}
\hline Axial Displacement $(\mu \mathrm{m})$ & $\boldsymbol{H}$ Value \\
\hline 0 & 20.40 \\
100 & 20.89 \\
200 & 21.43 \\
300 & 22.05 \\
400 & 22.61 \\
500 & 23.88 \\
600 & 24.84 \\
700 & 26.13 \\
800 & 27.48 \\
900 & 28.97 \\
1000 & 30.33 \\
1100 & 31.93 \\
1200 & 33.58 \\
1300 & 35.34 \\
1400 & 36.57 \\
1500 & 38.60 \\
1600 & 40.90 \\
1700 & 43.50 \\
1800 & 46.73 \\
1900 & 50.65 \\
2000 & 55.12 \\
2100 & 60.77 \\
2200 & 65.57 \\
\hline
\end{tabular}

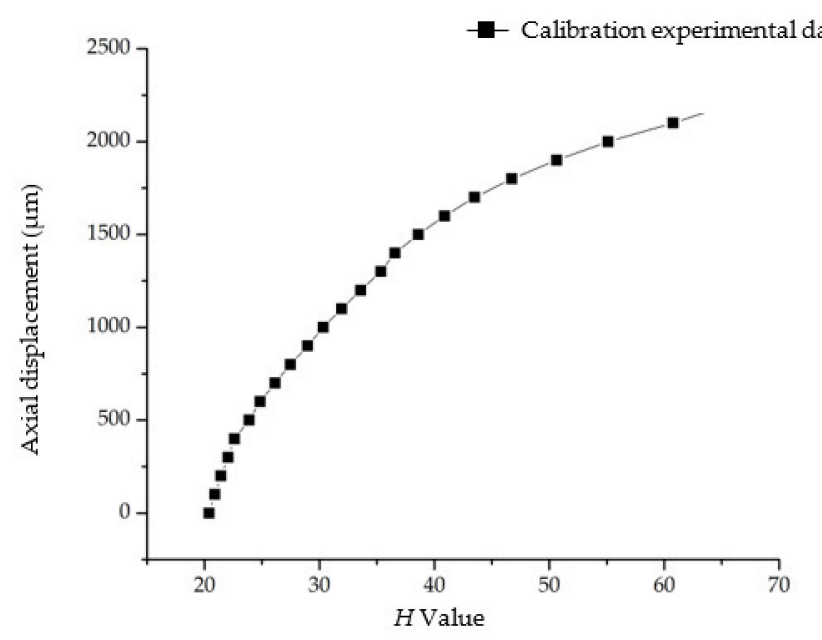

(a)

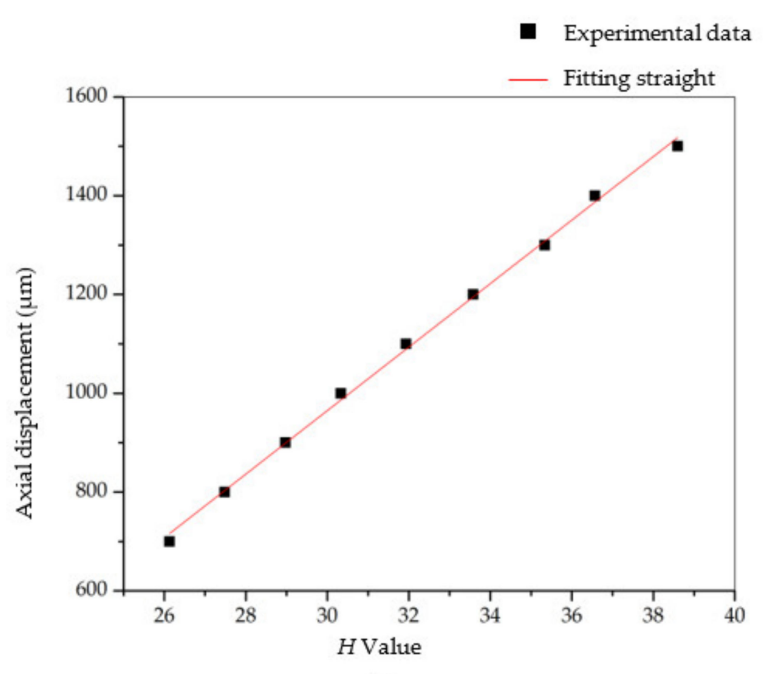

(b)

Figure 11. (a) Calibration experiment result. (b) Linear fitting result.

In the linear fitting, the standard square value of the fitting curve was 0.99 , and the relationship between the axial displacement of the sample $f(H)$ and the $H$ value is as below:

$$
f(H)=64.30 H-964.09
$$

\section{Error Theory Analysis and Correction}

\subsection{Error Theory Analysis}

In Section 2.3, the thickness calculation model of the transparent specimen is derived. The thickness of the transparent specimen depends on multiple parameters, such as the wavelength value of the light beams focused on the upper and the lower surfaces, the incidence angle $\theta$ and the conic focusing angle $\delta$. The angle incidence $\theta$ is a constant in 
this system. However, as the sample moves within the measurement range, the wavelength value of the light beams focused on the upper and lower surfaces and the angle $\delta$ change gradually.

Figure 12 shows the scenario that the sample is measured in different positions. When the sample is at position 1 , the wavelength value of the light beams focused on the upper and lower surfaces are $\lambda_{0}$ and $\lambda_{\mathrm{i}}$. When the sample moves to position 2 , the wavelength value of the focused light becomes $\lambda_{0}{ }^{\prime}$ and $\lambda_{\mathrm{i}}{ }^{\prime}$. The conic focusing angle $\delta$ becomes to a smaller angle $\delta^{\prime}$. According to Equation (15), the thickness $d$ will change as the wavelength value and the focusing angle $\delta$ have changed.

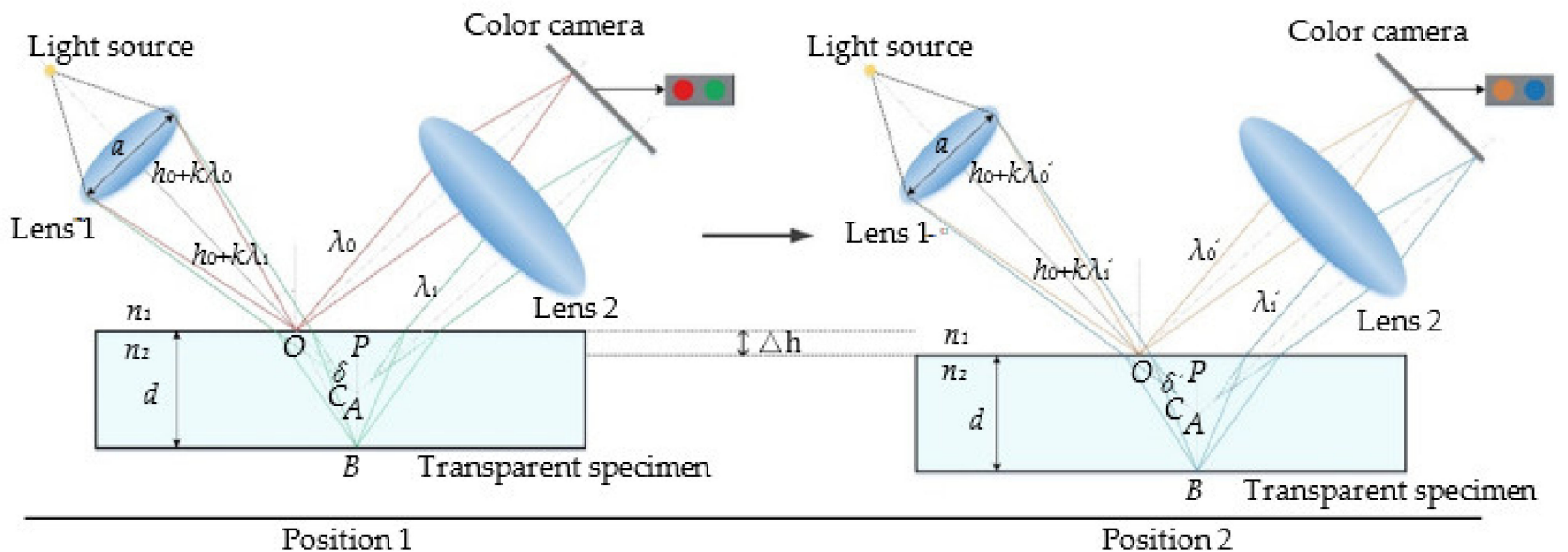

Figure 12. The light reflected from the upper and lower surfaces of the transparent sample in different positions.

In order to further study the correctness and feasibility of the error theory as mentioned above, it is needed further validation by simulated analysis. During the simulation, the thickness $d$ is set as $175.00 \mu \mathrm{m}$. The settings of other parameters are shown in Table 3 .

Table 3. The setting of parameters.

\begin{tabular}{cc}
\hline Parameter & Value \\
\hline$a(\mu \mathrm{m})$ & 9000.00 \\
$h_{0}(\mu \mathrm{m})$ & $10,000.00$ \\
$\delta\left(^{\circ}\right)$ & 20.00 \\
$\theta\left(^{\circ}\right)$ & 43.00 \\
$k$ & 64.30 \\
$n_{1}$ & 1.00 \\
$n_{2}$ & 1.50 \\
\hline
\end{tabular}

Equation (17), derived from Equation (15), can be seen as the functional relation between $H_{0}$ and $H_{\mathrm{i}}$ :

$$
H_{\mathrm{i}}=H_{0}+\frac{d\left(\tan \alpha^{\prime}-\tan \beta^{\prime}\right)}{k \cos \theta(\tan \alpha-\tan \beta)}
$$

Equation (17) shows that $H_{0}$ and $H_{\mathrm{i}}$ are approximately linear.

Since the angle $\delta$ is a variable value, the constant terms in Equation (17) do not remain constant. Using the data in Table 3 to simulate Equation (17), the result is shown in Figure 13. 


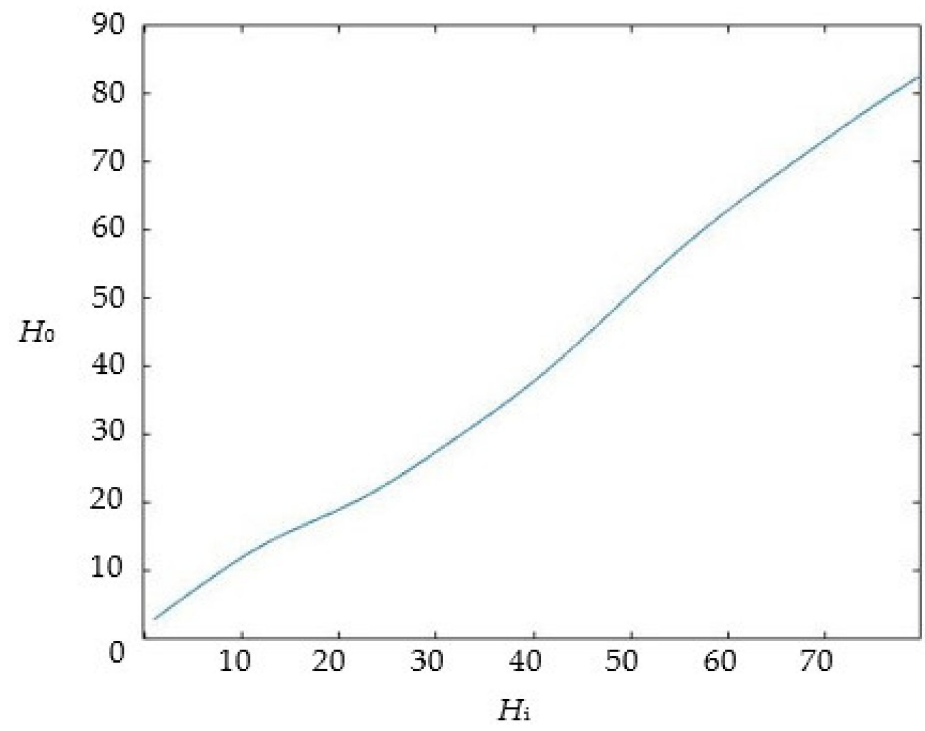

Figure 13. The relationship between $H_{0}$ and $H_{\mathrm{i}}$.

The following formula can be obtained by linear fitting the data in Figure 13:

$$
H_{0}=1.05 H_{\mathrm{i}}-4.18
$$

This shows that the $H$ values of the light beams focused on the upper and lower surfaces have a good linearity when the sample is at different positions.

In order to verify the difference of measurement results in different positions, the thickness $d$ in Equation (15) is simulated, as shown in Figure 14. To indicate that the sample is in different positions, the value of $H_{0}$ is set from 0 to 80 . The value of $H_{\mathrm{i}}$ can be calculated by Equation (18) and other parameters in Table 3.

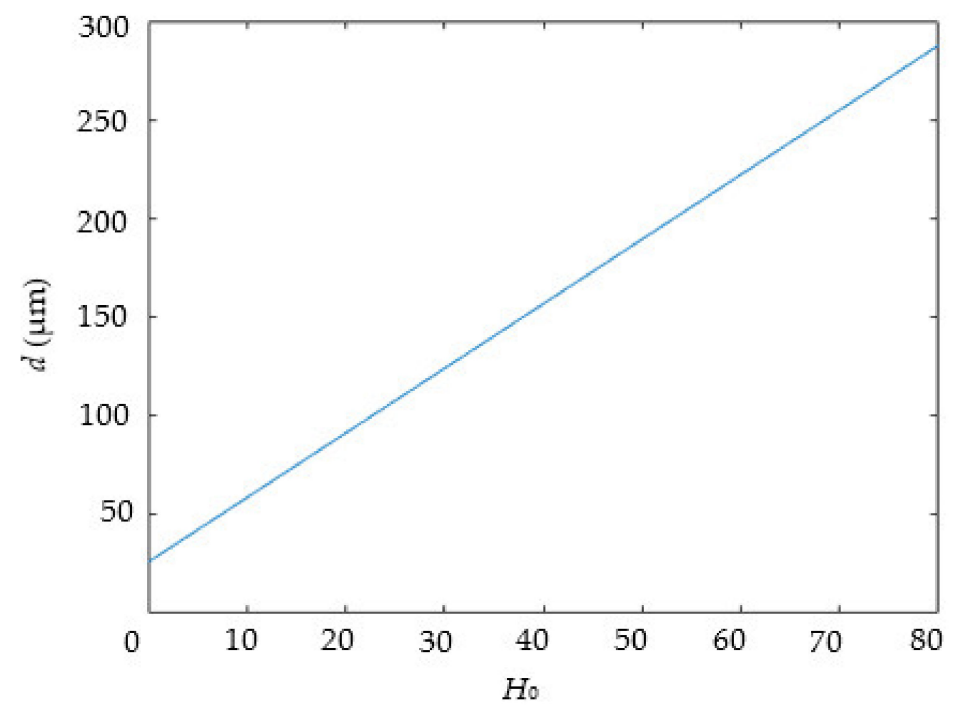

Figure 14. The variation of thickness $d$ in different positions.

The simulation result in Figure 14 shows that the thickness $d$ gradually decreases as $H_{0}$ increases; in other words, as the sample moves away from the incident light. This simulation result shows there is certain measurement error in different positions. The thickness $d$ is also related to the angle $\delta$. Then, Equation (10) is simulated as parameters set according to Table 3. 
It can be seen from Figure 15 that the angle $\delta$ gradually decreases when the sample moves away from the incident light, which is consistent with the geometric analysis result in Figure 12.

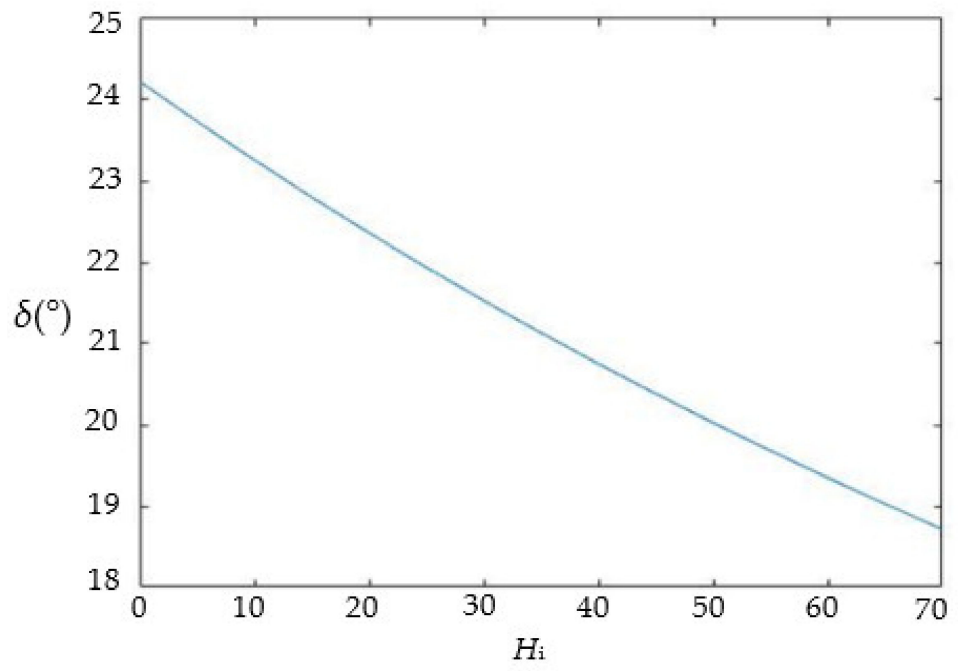

Figure 15. The variation of angle $\delta$ in different positions.

\subsection{Error Correction}

According to the error theoretical analysis in Section 4.1, the measurement error of the thickness $d$ comes from the change of the angle $\delta$ in different positions. Therefore, accurately determining the value of the angle $\delta$ in different positions is the key to improving the precision of the system, reducing the error and achieving measurement in different positions.

In Section 2.3, the axial position has a linear relationship with the wavelength value of the focused light and the wavelength value can be converted to $H$ value. Then, Equation (10) can be expressed as:

$$
\delta=\arctan \left[\frac{a}{2\left(h_{0}+k_{1} H_{\mathrm{i}}\right)}\right]
$$

The angle $\delta$ is related to the $h_{0}$ and $H_{\mathrm{i}}$, and the values of the angle $\delta$ and the $H_{\mathrm{i}}$ will change as the sample moving. Therefore, it is necessary to determine the accurate value of $h_{0}$ and the value of the angle $\delta$ in different positions.

The specific steps of the error correction method are as follows:

1. After the calibration experiment, the measurement range of the system can be represented by a range of $H$ values. A transparent specimen with known thickness was placed in the system. The upper surface was placed accurately at the starting position of the measurement range and the lower surface must also be within the measurement range. Height values of the light beams focused on the upper and lower surfaces were recorded as $H_{0}$ and $H_{\mathrm{i}}$.

2. The values of $d, H_{0}$ and $H_{\mathrm{i}}$ were taken into Equations (10) and (15) to calculate the angle $\delta$ in this position.

3. The angle $\delta$ calculated in step 2 was taken into Equation (19) to find $h_{0}$ which was set as the original parameters of the system.

4. When measuring in different positions, the $H_{\mathrm{i}}$ value of the light beams focused on the lower surface in the current position was recorded. The angle $\delta$ of the current position then was calculated using Equation (19).

5. The thickness in each position was calculated by using the data of the $H_{0}, H_{\mathrm{i}}$ and $\delta$ in the current position. 


\section{Thickness Measurement Experiments of the Transparent Specimens}

In Section 2.2, the calculation formula for the thickness of the transparent specimen has been proposed. This section will provide the experimental details in this study. Using the experimental platform shown in Figure 9, the calibration work was performed. Then, three kinds of transparent specimens with different thicknesses and refractive indexes were measured. The physical images of Sample 1, Sample 2, Sample 3 and Sample 4 are shown in Figure 16. Sample 1 was used to calculate the initial parameters and angles of the system, and Sample 2, 3 and 4 were used as experimental samples to measure the thickness with the error correction model. Sample 2 is made of glass, Sample 3 is made of PVC and Sample 4 is made of PET. Additionally, the true thickness values of Samples 2, 3 and 4 are $172.54 \mu \mathrm{m}, 679.72 \mu \mathrm{m}$ and $245.00 \mu \mathrm{m}$, respectively, which are measured by a universal length measuring machine and its product model is TRIMOS LABC500. The universal length measuring machine is shown in Figure 17.

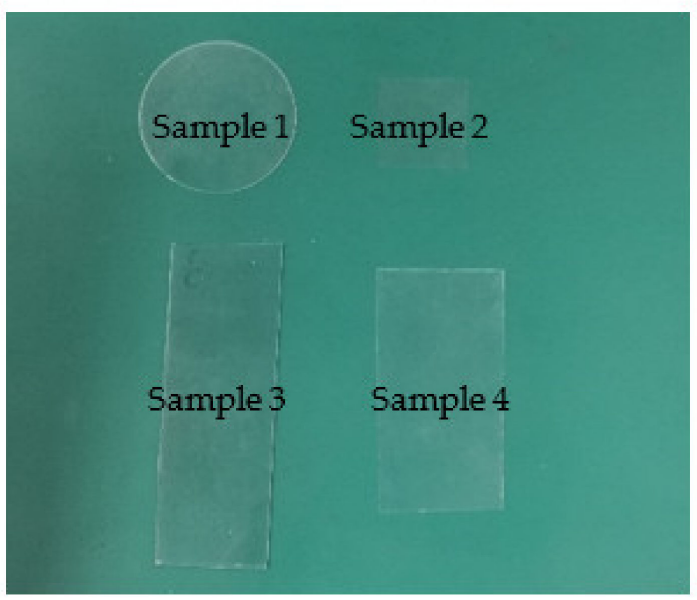

Figure 16. Picture of the transparent specimens.

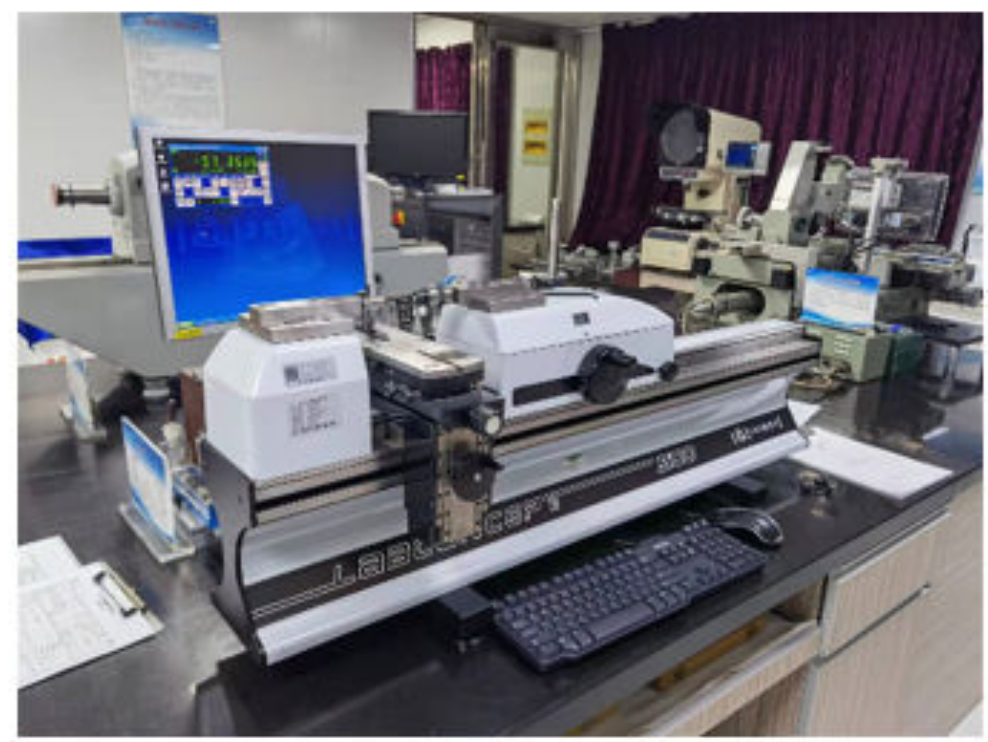

Figure 17. Picture of the TRIMOS LABC500.

Firstly, according to the error theoretical mentioned in Section 4.2, Sample 1 was selected to determine the initial parameter $h_{0}$ of the system. Sample 1 was placed in the starting position within the linear range of the system. The parameters of the system are listed in Table 4. 
Table 4. Fixed parameters in the system.

\begin{tabular}{cc}
\hline Parameter & Value \\
\hline$\theta\left(^{\circ}\right)$ & 43.00 \\
$\delta_{0}\left(^{\circ}\right)$ & 30.89 \\
$h_{0}(\mu \mathrm{m})$ & 5695.53 \\
\hline
\end{tabular}

Then, the thicknesses of Samples 2, 3 and 4 were measured, respectively, and the experiment of each sample was repeated 15 times in different positions within the measurement range. The measurement data of Samples 2, 3 and 4 were listed in the following tables. In each table, the measurement data with and without correction were provided (The thicknesses without correction were calculated through the angle $\delta_{0}$ in Table 4).

\subsection{Glass Specimen}

When using glass such as Samples 2 as the specimen, the value of refractive index $n_{2}$ is 1.50 .

Figure 18 shows one of the 15 captured pictured in the measurement of Sample 2. Using the color conversion algorithm, the $R, G$, and $B$ information of these 15 pieces of pictures were converted to $H$ values. According to the correction method in Section 4.2, the angle $\delta$ was calculated and thickness $d$ was calculated according to Equations (8) and (13), as listed in Table 5.

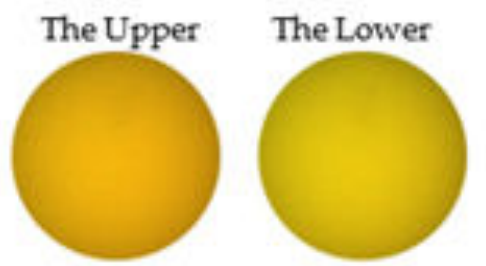

Figure 18. Experimental picture of Sample 2.

Table 5. Experimental data of the thickness of Sample 2.

\begin{tabular}{|c|c|c|c|c|c|c|}
\hline $\begin{array}{l}\text { Position } \\
\text { Number }\end{array}$ & $\begin{array}{c}H \text { Value of } \\
\text { Upper Surface }\end{array}$ & $\begin{array}{c}H \text { Value of } \\
\text { Lower Surface }\end{array}$ & $\begin{array}{l}\text { Value of } \\
\text { Difference }\end{array}$ & Angle $\delta\left(^{\circ}\right)$ & $\begin{array}{l}\text { Thickness without } \\
\text { Correction } d^{\prime}(\mu \mathrm{m})\end{array}$ & $\begin{array}{l}\text { Thickness with } \\
\text { Correction } d(\mu \mathrm{m})\end{array}$ \\
\hline 1 & 27.64 & 28.43 & 0.79 & 30.88 & 174.99 & 174.93 \\
\hline 2 & 28.21 & 29.00 & 0.79 & 30.76 & 174.51 & 173.43 \\
\hline 3 & 28.46 & 29.27 & 0.81 & 30.70 & 178.48 & 176.90 \\
\hline 4 & 29.32 & 30.13 & 0.81 & 30.52 & 177.91 & 174.82 \\
\hline 5 & 29.56 & 30.38 & 0.82 & 30.47 & 180.65 & 177.07 \\
\hline 6 & 29.95 & 30.76 & 0.81 & 30.39 & 178.01 & 173.84 \\
\hline 7 & 30.33 & 31.16 & 0.83 & 30.30 & 181.19 & 176.27 \\
\hline 8 & 30.68 & 31.50 & 0.82 & 30.23 & 180.52 & 175.05 \\
\hline 9 & 31.53 & 32.36 & 0.83 & 30.06 & 182.88 & 175.92 \\
\hline 10 & 31.95 & 32.80 & 0.85 & 29.97 & 186.11 & 178.30 \\
\hline 11 & 32.60 & 33.43 & 0.83 & 29.84 & 184.25 & 175.51 \\
\hline 12 & 33.79 & 34.64 & 0.85 & 29.60 & 187.55 & 176.76 \\
\hline 13 & 34.56 & 35.42 & 0.86 & 29.44 & 190.69 & 178.52 \\
\hline 14 & 35.64 & 36.51 & 0.87 & 29.23 & 192.90 & 178.95 \\
\hline 15 & 36.83 & 37.70 & 0.87 & 28.99 & 192.40 & 176.77 \\
\hline \multirow{2}{*}{\multicolumn{2}{|c|}{$\begin{array}{l}\text { The average value of the } \\
\text { thickness with correction }(\mu \mathrm{m})\end{array}$}} & \multirow{2}{*}{\multicolumn{2}{|c|}{176.20}} & \multirow{2}{*}{\multicolumn{2}{|c|}{$\begin{array}{l}\text { Standard deviation } \sigma \text { of } d^{\prime}(\mu \mathrm{m}) \\
\text { Standard deviation } \sigma \text { of } d(\mu \mathrm{m})\end{array}$}} & 5.97 \\
\hline & & & & & & 1.64 \\
\hline
\end{tabular}

The thickness measurement data of Sample 2 was shown in Table 5. 
The calculation formula of the standard deviation $\sigma$ is:

$$
\sigma=\sqrt{\frac{\sum\left(x_{i}-\bar{x}\right)^{2}}{n-1}}
$$

Figure 19 showed the measurement results of Samples 2. It was obviously that the measurement results with correction had a smaller error than those without correction.

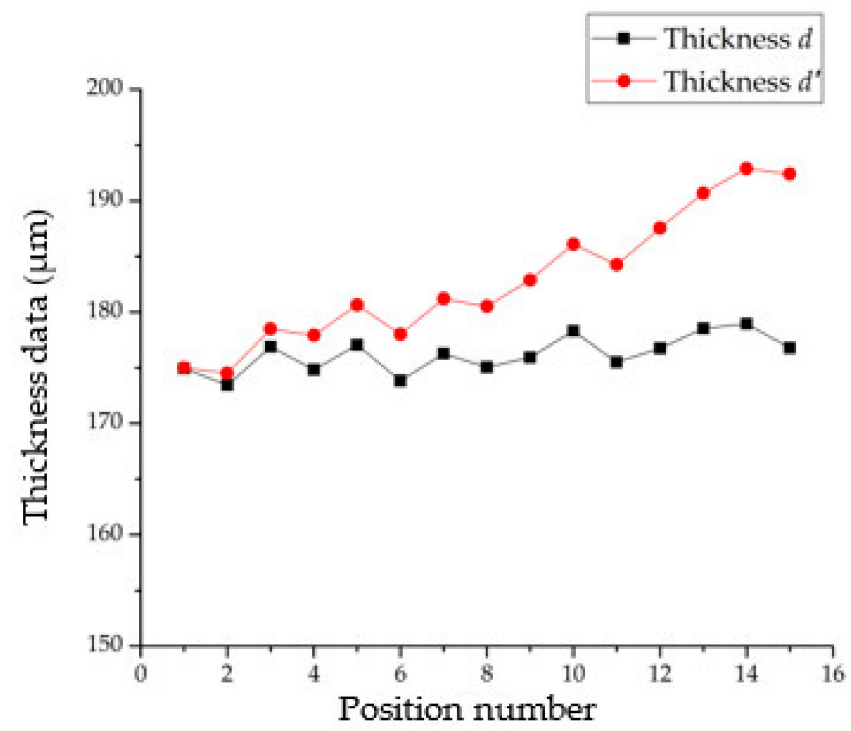

Figure 19. The thickness measurement data of Sample 2.

With correction, the thickness of Sample 2 was $176.26 \mu \mathrm{m}$ and the repeatability decreased from $11.94 \mu \mathrm{m}$ to $3.28 \mu \mathrm{m}$. The measurement error was $2.12 \%$.

\subsection{PVC Specimen}

When using PVC such as Sample 3 as the specimen, the value of refractive index $n_{2}$ is 1.55. Additionally, the thickness measurement data and result of Sample 3 are shown in Table 6 and Figure 20, respectively.

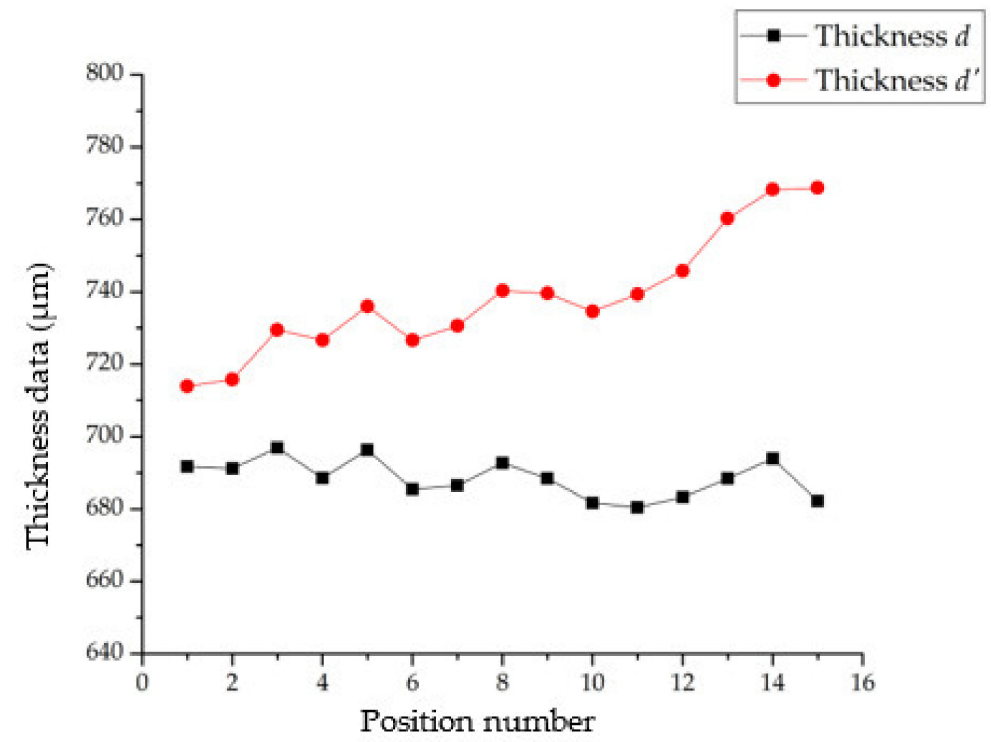

Figure 20. The thickness measurement data of Sample 3. 
Table 6. Experimental data of the thickness of Sample 3.

\begin{tabular}{|c|c|c|c|c|c|c|}
\hline $\begin{array}{l}\text { Position } \\
\text { Number }\end{array}$ & $\begin{array}{c}H \text { Value of } \\
\text { Upper Surface }\end{array}$ & $\begin{array}{c}H \text { Value of } \\
\text { Lower Surface }\end{array}$ & $\begin{array}{c}\text { Value of } \\
\text { Difference }\end{array}$ & Angle $\delta\left(^{\circ}\right)$ & $\begin{array}{l}\text { Thickness without } \\
\text { Correction } d^{\prime}(\mu \mathrm{m})\end{array}$ & $\begin{array}{l}\text { Thickness with } \\
\text { Correction } d(\mu \mathrm{m})\end{array}$ \\
\hline 1 & 27.80 & 30.83 & 3.03 & 30.37 & 713.92 & 691.77 \\
\hline 2 & 28.16 & 31.19 & 3.04 & 30.30 & 715.81 & 691.18 \\
\hline 3 & 29.23 & 32.33 & 3.10 & 30.06 & 729.44 & 696.87 \\
\hline 4 & 30.13 & 33.22 & 3.09 & 29.88 & 726.65 & 688.55 \\
\hline 5 & 30.26 & 33.38 & 3.12 & 29.85 & 735.97 & 696.35 \\
\hline 6 & 30.62 & 33.71 & 3.09 & 29.78 & 726.60 & 685.50 \\
\hline 7 & 31.07 & 34.17 & 3.10 & 29.69 & 730.59 & 686.43 \\
\hline 8 & 31.51 & 34.65 & 3.14 & 29.59 & 740.35 & 692.71 \\
\hline 9 & 32.11 & 35.25 & 3.14 & 29.47 & 739.57 & 688.46 \\
\hline 10 & 32.51 & 35.63 & 3.12 & 29.40 & 734.63 & 681.64 \\
\hline 11 & 33.46 & 36.60 & 3.14 & 29.21 & 739.28 & 680.39 \\
\hline 12 & 34.01 & 37.18 & 3.17 & 29.09 & 745.83 & 683.23 \\
\hline 13 & 35.41 & 38.64 & 3.23 & 28.82 & 760.31 & 688.49 \\
\hline 14 & 35.71 & 38.97 & 3.26 & 28.75 & 768.28 & 693.92 \\
\hline 15 & 38.06 & 41.32 & 3.26 & 28.31 & 768.72 & 682.25 \\
\hline \multirow{2}{*}{\multicolumn{5}{|c|}{ The average value of the thickness with correction $(\mu \mathrm{m})$}} & $\begin{array}{c}\text { Standard deviation } \\
\sigma \text { of } d^{\prime}(\mu \mathrm{m})\end{array}$ & 16.69 \\
\hline & & & & & $\begin{array}{c}\text { Standard deviation } \\
\sigma \text { of } d(\mu \mathrm{m})\end{array}$ & 5.27 \\
\hline
\end{tabular}

With correction, the thickness of Sample 3 was $688.52 \mu \mathrm{m}$ and the repeatability decreased from $33.38 \mu \mathrm{m}$ to $10.54 \mu \mathrm{m}$. The measurement error was $1.29 \%$.

\subsection{PET Specimen}

When using PET such as Sample 4 as the specimen, the value of refractive index $n_{2}$ is 1.65. The thickness measurement data and result of Sample 4 are shown in Table 7 and Figure 21, respectively.

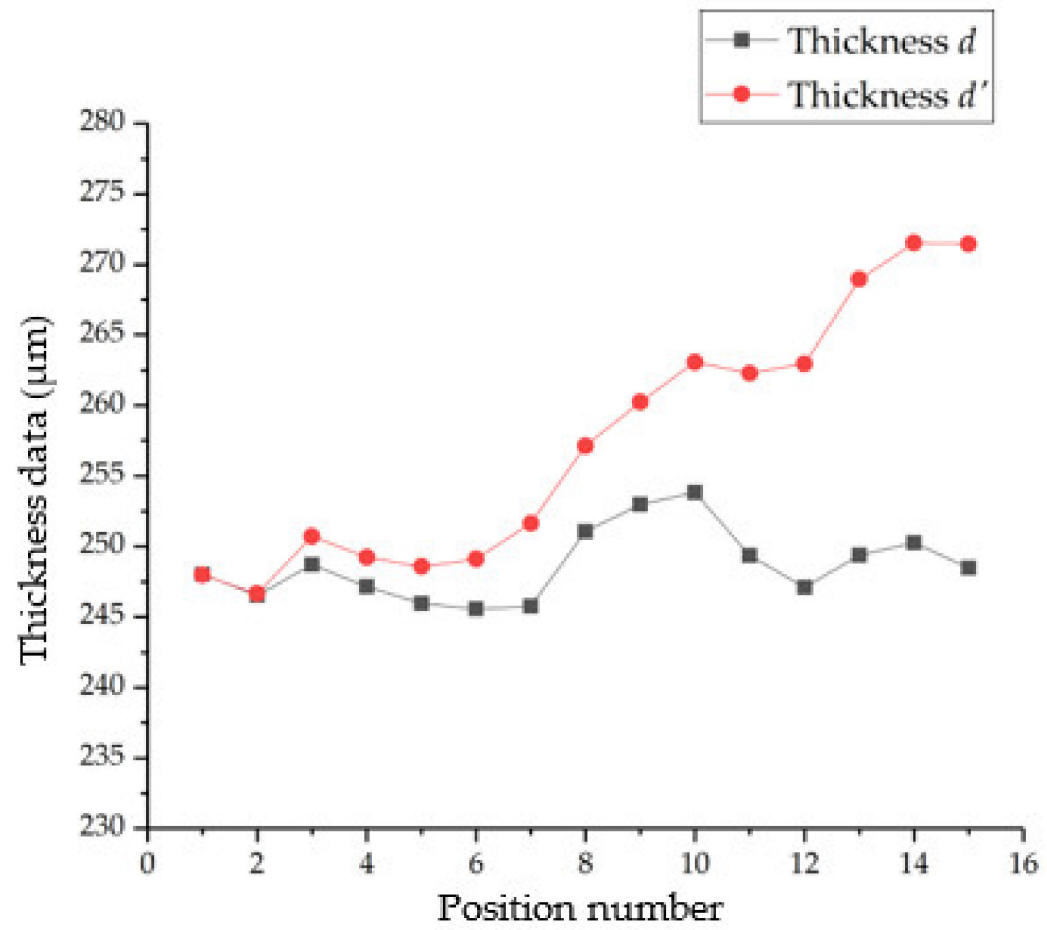

Figure 21. The thickness measurement data of Sample 4 . 
Table 7. Experimental data of the thickness of Sample 4.

\begin{tabular}{ccccc}
\hline Position Number & $\begin{array}{c}\boldsymbol{H} \text { Value of Upper } \\
\text { Surface }\end{array}$ & $\begin{array}{c}\boldsymbol{H} \text { Value of Lower } \\
\text { Surface }\end{array}$ & $\begin{array}{c}\text { Thickness without } \\
\text { Correction } \boldsymbol{d}^{\prime}(\boldsymbol{\mu m})\end{array}$ & $\begin{array}{c}\text { Thickness with } \\
\text { Correction } \boldsymbol{d}(\boldsymbol{\mu} \text { m) })\end{array}$ \\
\hline 1 & 28.54 & 29.50 & 247.99 & 248.02 \\
2 & 28.60 & 29.56 & 246.65 & 246.54 \\
3 & 29.25 & 30.22 & 250.71 & 248.72 \\
4 & 29.30 & 30.26 & 249.25 & 247.16 \\
5 & 29.49 & 30.45 & 248.57 & 245.57 \\
6 & 29.83 & 30.79 & 249.11 & 245.77 \\
7 & 30.68 & 31.66 & 251.65 & 251.05 \\
8 & 30.70 & 31.69 & 257.14 & 252.99 \\
9 & 31.08 & 32.09 & 260.23 & 253.83 \\
10 & 31.80 & 32.82 & 262.08 & 249.37 \\
11 & 33.26 & 34.28 & 262.96 & 247.11 \\
12 & 34.46 & 35.48 & 268.95 & 249.39 \\
13 & 35.86 & 36.89 & 271.52 & 250.26 \\
14 & 36.51 & 37.56 & 271.46 & 248.48 \\
15 & 37.28 & 38.34 & & 8.95 \\
\hline
\end{tabular}

With correction, the thickness of Sample 4 was $248.68 \mu \mathrm{m}$ and the repeatability decreased from $17.90 \mu \mathrm{m}$ to $5.06 \mu \mathrm{m}$. The measurement error was $1.50 \%$.

As shown in Figures 19-21, when the Samples were placed at different positions, the measurements without correction showed increasing measurement errors, leading to a significant measurement uncertainty (represented by standard deviation). In contrast, when the proposed correction method was applied, the measurement results showed significantly improved position independence. At the same time, the correction method has good correction effect on the samples with different refractive indexes.

\section{Discussion}

1. Experimental results showed that axial measurement accuracy of the system could reach the micron level and the measurement errors were significantly reduced, as compared to the uncorrected system.

2. The relationship between the thickness $d$ and the angle $\delta$ was discussed and derived in this paper. We calculated the value of $h_{0}$ of the system, which was $5695.53 \mu \mathrm{m}$.

3. It was found that the parameters of the system were inconsistent when the transparent specimen at different positions. Additionally, an error correction model was proposed by calculating the parameter values.

4. The transparent specimens used in this experiment were made of different materials such as glass, PVC and PET. Additionally, a series of experiments were conducted using the error correction model.

\section{Conclusions}

In the fields of thickness measurement of transparent specimen, the chromatic confocal system with inclined illumination is able to achieve better signal-to-noise ratio. However, the measurement results at different axial positions vary significantly.

In this paper, position-induced error was analyzed. It was found that the parameters of the confocal model are inconsistent when the sample is axially moving. Based on this finding, an error correction model was proposed by calculating the parameter values at different positions. Using this error correction, a series of experiments were conducted with transparent specimens made of different materials such as glass, PVC, and PET. The experimental results showed that the correction could effectively correct the measurement error induced by the position change. Therefore, the measurement accuracy can be effectively improved. 


\begin{abstract}
Author Contributions: Conceptualization, Q.Y. and C.W.; methodology, Q.Y.; software, C.W.; validation, C.W., Y.Z. and S.H.; formal analysis, C.W. and S.H.; investigation, Y.W.; resources, L.X.; data curation, F.C.; writing—original draft preparation, C.W.; writing—review and editing, T.L. (Ting Liu) and T.L. (Tianliang Lin). All authors have read and agreed to the published version of the manuscript.

Funding: This research was funded by the National Natural Science Foundation of China (No. 52075190 and No. 62075067); the Collaborative Innovation Platform OF Fuzhou-Xiamen-Quanzhou Independent Innovation Demonstration Area, China (No. 3502ZCQXT202002); the Science of Technology Program of Fujian, China (No.2019I0013); and the Open Research Fund of Anhui Engineering Technology Research Center of Automotive New Technique (No. QCKJ202006).
\end{abstract}

Institutional Review Board Statement: Not applicable.

Informed Consent Statement: Not applicable.

Data Availability Statement: Data sharing is not applicable to this article.

Conflicts of Interest: The authors declare no conflict of interest.

\title{
References
}

1. Wang, Y.; Xi, M.; Liu, H.; Ding, Z.; Du, W.; Meng, X.; Sui, Y.; Li, J.; Jia, Z. On-machine noncontact scanning of high-gradient freeform surface using chromatic confocal pron diamond turning machine. Opt. Laser Technol. 2021, 134, 106569. [CrossRef]

2. Chen, Z.; Wang, Z.; Ren, M.; Zhang, X.; Zhu, L.; Jiang, X. Development of an on-machine measurement system for ultra-precision mechine tools using a chromatic confocal sensor. Precis. Eng. 2022, 74, 232-241. [CrossRef]

3. Chen, C.C.A.; Li, J.; Liao, W.; Ciou, Y.; Chen, C. Dynamic pad surface metrology monitoring by swing-arm chromatic confocal system. Appl. Sci. 2021, 14, 179. [CrossRef]

4. Zhou, R.; Shen, D.; Huang, P.; Kong, L.; Zhu, Z. Chromatic confocal sensor-based sub-aperture scanning and stitching for the measurement of microstructured optical surfaces. Opt. Express 2021, 29, 33512-33526. [CrossRef] [PubMed]

5. Yang, X.; Wang, Y.; Zhang, H.; Qin, H.; Wang, S.; Tong, K.; Zhou, K.; Sun, R.; Yue, S.; Chen, X.; et al. Fiber-optic large-depth 3D chromatic confocal endomicroscopy. Biomed. Opt. Express 2022, 13, 300-313. [CrossRef]

6. Kulkarni, N.; Masciola, A.; Nishant, A.; Kim, K.; Choi, H.; Gmitro, A.; Freeman, E.; Semeere, A.; Nakalembe, M.; Kang, D. Low-cost, chromatic confocal endomicroscope for cellular imaging in vivo. Biomed. Opt. Express 2021, 12, 5629-5643. [CrossRef]

7. Gao, Y. 3D reconstruction of aero engine blade detection. Sci. Technol. Innov. Her. 2020, 17, 52-53.

8. Gao, Y. Characterization of chemical mechanical planarization with white light in interferometry. Equip. Electron. Prod. Manuf. 2006, 10, 19-23.

9. Duque, D.; Garzón, J.; Gharbi, T. A study of dispersion in chromatic confocal microscopy using digital image processing. Opt. Laser Technol. 2020, 131, 106414. [CrossRef]

10. Ma, J.; Qi, Y.; Lu, Z.; Su, J.; Yang, G.; Qi, W.; Zhang, Q.; Chen, J. Design of linear dispersive objective for chromatic confocal displacement sensor. Chin. J. Lasers 2019, 46, 219-225.

11. Bai, J.; Li, X.; Zhou, Q.; Ni, K.; Wang, X. Improved chromatic confocal displacement-sensor based on a spatial-bandpass-filter and an X-shaped fiber-coupler. Opt. Express 2019, 27, 10961-10973. [CrossRef]

12. Bai, J.; Wang, X.; Li, X.; Zhou, Q.; Ni, K. Chromatic confocal displacement sensor with optimized dispersion probe and modified centroid peak extraction algorithm. Sensors 2019, 19, 3592. [CrossRef]

13. Chen, C.; Yang, W.; Wang, J.; Lu, W.; Liu, X.; Jiang, X. Accurate and efficient height extraction in chromatic confocal microscopy using corrected fitting of the differential signal. Precis. Eng. 2019, 56, 447-454. [CrossRef]

14. Chen, C.; Leach, R.; Wang, J.; Liu, X.; Jiang, X.; Lu, W. Two-dimensional spectral signal model for chromatic confocal microscopy. Opt. Express 2021, 29, 7179-7196. [CrossRef]

15. Bai, J.; Li, X.; Wang, X.; Ni, K.; Zhou, Q. Self-reference dispersion correction for chromatic confocal displacement measurement. Opt. Lasers Eng. 2021, 140, 106540. [CrossRef]

16. Yu, Q.; Zhang, K.; Cui, C.; Zhou, R.; Cheng, F.; Ye, R.; Zhang, Y. Method of thickness measurement for transparent specimens with chromatic confocal microscopy. Appl. Opt. 2018, 57, 9722-9728. [CrossRef]

17. Li, J.; Zhao, Y.; Du, H.; Zhu, X.; Wang, K.; Zhao, M. Adaptive modal decomposition based overlapping-peaks extraction for thickness measurement in chromatic confocal microscopy. Opt. Express 2020, 28, 36176-36187. [CrossRef]

18. Li, J.; Zhu, X.; Du, H.; Ji, Z.; Wang, K.; Zhao, M. Method of thickness measurement for self-supporting film with double chromatic confocal probes. Appl. Opt. 2021, 60, 9447-9452. [CrossRef]

19. Berkovic, G.; Zilberman, S.; Shafir, E.; Rubin, D. Chromatic confocal displacement sensing at oblique incidence angles. Appl. Opt. 2020, 59, 3183-3186. [CrossRef]

20. Yu, Q.; Zhang, Y.; Shang, W.; Dong, S.; Wang, C.; Wang, Y.; Liu, T.; Cheng, F. Thickness Measurement for Glass Slides Based on Chromatic Confocal Microscopy with Inclined Illumination. Photonics 2021, 8, 170. [CrossRef] 
21. Yu, Q.; Zhang, Y.; Zhang, Y.; Cheng, F.; Shang, W.; Wang, Y. A novel chromatic confocal one- shot 3D measurement system based on DMD. Measurement 2021, 186, 110140. [CrossRef]

22. Miks, A.; Novak, J.; Novak, P. Analysis of method for measuring thickness of plane-parallel plates and lenses using chromatic confocal sensor. Appl. Opt. 2010, 47, 3259-3264. [CrossRef] 